\title{
Femtosecond Frequency Conversion in Diamond under Gaussian and Bessel Beam Pumping
}

\author{
Vasili G. Savitski ${ }^{1}$, Rolf B. Birch ${ }^{1}$, Sean Reilly ${ }^{1}$, Vladimir L. Kalashnikov ${ }^{2}$, Alan J. Kemp \\ ${ }^{1}$ Institute of Photonics, Dept. of Physics, University of Strathclyde, Glasgow, Scotland, G1 1RD \\ ${ }^{2}$ Institute of Photonics, Vienna University of Technology, Vienna, Austria, A-1040
}

Diamond Raman lasers (DRLs) have been the subject of extensive research in the recent years. Continuouswave and pulsed, intra- and extra-cavity DRLs emitting from UV to IR have been demonstrated [1]. The majority of these studies were carried out in the steady-state mode, when the pump pulse duration is longer than the dephasing time in diamond. Less work has been done on the transient mode, when the pulse duration is shorter than the dephasing time: DRLs under femtosecond (fs) pumping in synchronously-pumped cavities has been demonstrated [2], and supercontinuum (SC) generation in diamond under fs pumping has been reported [3]. The major mechanism for SC generation under fs laser pumping is believed to be self-phase modulation (SPM). It has been shown before that this effect can be significantly reduced when using the Bessel beam pumping. In this work we present a first comparison study of the effect of Gaussian and Bessel pump beams on the spectral properties of nonlinear frequency conversion in diamond in transient mode.

Laser pulses at $800 \mathrm{~nm}$ with duration of $150 \mathrm{fs}$ with the maximum pulse energy of $1 \mathrm{~mJ}$ were used as a pump. An uncoated diamond crystal with a length of $6.5 \mathrm{~mm}$ along the $\langle 110\rangle$ axis and an absorption coefficient at $1064 \mathrm{~nm}$ measured to be $\sim 0.03 \mathrm{~cm}^{-1}$ was placed either at the focus of a $50 \mathrm{~mm}$ focal length spherical lens or $\sim 1.3$ $\mathrm{cm}$ from the tip of an axicon with $20^{\circ}$ wedge angle. The pump spot diameter of the Gaussian / Bessel beam was calculated to be $\sim 5 \mu \mathrm{m} / \sim 3.6 \mu \mathrm{m}$ in each case. Pulse energy of the output was measured after a long pass filter with a cut-on wavelength of $850 \mathrm{~nm}$.

Under Gaussian beam pumping SC generation was observed, similar to the results in [3], with the cut off wavelength of $\sim 525 \mathrm{~nm}$ (Fig. 1 (a)). On the long wavelength side the measurements were limited to $1025 \mathrm{~nm}$ by the available spectrometer. A peak, corresponding to the $1^{\text {st }}$ Raman Stokes at $\sim 895 \mathrm{~nm}$, appeared in the emission spectra at pump pulse energies $>2.5 \mu \mathrm{J}$. The output pulse energy had an almost linear dependence on the pump energy (Fig. 1 (a), inset).

In case of Bessel beam pumping the output spectrum consisted mainly of Raman Stokes and anti-Stokes lines (Fig. $1(\mathrm{~b})) .1^{\text {st }}$ and $2^{\text {nd }}$ Stokes lines were concentrated in the centre of the beam emitted by the diamond, while anti-Stokes emission propagated in the first and second rings. The output pulse energy saturated at $\sim 55 \mu \mathrm{J}$ of pump pulse energy (Fig. 1 (b), inset). This dependence is similar to the one observed under the impulsive stimulated Raman scattering, when the pump pulse duration is shorter than the vibrational period in a crystal, although in our case the pulse duration was 6 times longer than the vibrational period in diamond.

Significantly lower conversion efficiencies of the pump emission with the Bessel beam $(0.6 \%)$ in comparison with that with the Gaussian beam $(\sim 33 \%)$ can be attributed to the fact that according to our calculations the central maximum of the Bessel beam contained only $0.3 \%$ of the total pump power. The difference in spectral characteristics of the output under Bessel beam pumping indicates reduced SPM, with the Raman effect being the major mechanism for frequency conversion. The results of numerical modelling will be presented.
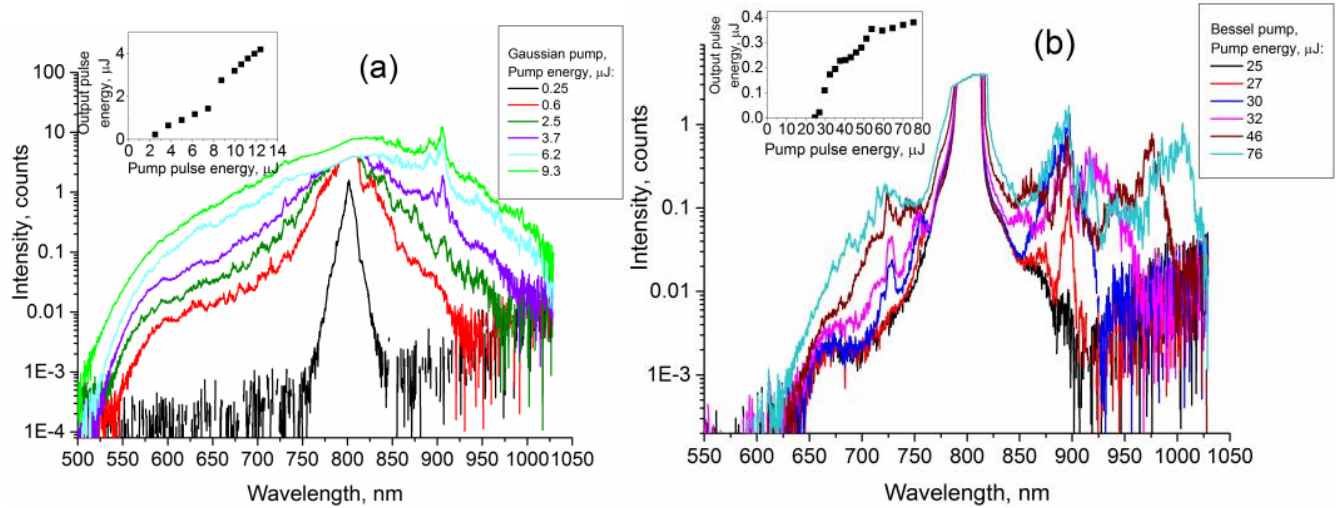

Fig. 1 Spectra of emission generation in diamond under Gaussian (a) and Bessel (b) beam pumping at $800 \mathrm{~nm}$ at different pump energies. Insets: output pulse energy of stimulated emission at the wavelengths longer than $850 \mathrm{~nm}$ as a function of pump pulse energy.

\section{References}

[1] V. G. Savitski, I. Friel, J. E. Hastie, M. D. Dawson, D. Burns, A. J. Kemp, "Characterization of Single-Crystal Synthetic Diamond for Multi-Watt Continuous-Wave Raman Lasers," Quantum Electronics, IEEE Journal of 48, 328 (2012).

[2] M. Murtagh, J. Lin, J. Trägårdh, G. McConnell, D.J. Spence, "Ultrafast second-Stokes diamond Raman laser," Opt. Express 24, 8149

(2016).

[3] T. M. Kardaś, B. Ratajska-Gadomska, W. Gadomski, A. Lapini, and R. Righini, "The role of stimulated Raman scattering in supercontinuum generation in bulk diamond," Opt. Express 21, 24201 (2013). 